\title{
Protocol
}

\section{Single Cell/Cellular Subregion-Targeted Phototransfection}

\author{
Jaehee Lee, ${ }^{1}$ Jai-Yoon Sul, ${ }^{1}$ and James H. Eberwine ${ }^{1,2,3}$ \\ ${ }^{1}$ Department of Pharmacology, University of Pennsylvania, Philadelphia, Pennsylvania 19104; ${ }^{2}$ Penn Genome \\ Frontiers Institute, Philadelphia, Pennsylvania 19104-6108
}

\begin{abstract}
Introduction of exogenous genetic material, such as DNA, mRNA, siRNA, or miRNA, into cells is routinely performed using one of the many different standardized methods, including lipid-mediated transfection, electroporation, and microinjection to identify their biological function. The ability to control the location and amount of nucleic acids introduced into a cell is particularly important for studying polarized cells such as neurons. Lipid-mediated transfection is simple and fast but lacks regional specificity of delivery, whereas microinjection is regionally specific but labor intensive. To overcome these obstacles, we developed and use the method of phototransfection. In this method, a conventional microscope with a high-power pulse laser of any wavelength is able with minimal destructiveness to induce transient pores into the plasma membrane of the target cell, which remain open long enough to allow exogenous genetic material to diffuse into the cytosol before the pore closes due to membrane dynamics. The technique is not limited by choice of cell type or by genetic material to be introduced, and for RNA allows transfection of multiple mRNAs simultaneously in any desired amount or ratio. Further, phototransfection allows the target area to be a specific cell in a population of cells or a specific subregion within a cell. This protocol summarizes the key steps for performing phototransfection, provides a guide to optimization, and uses as an example green fluorescent protein (GFP) mRNA transfection within a single neuronal process.
\end{abstract}

It is essential that you consult the appropriate Material Safety Data Sheets and your institution's Environmental Health and Safety Office for proper handling of equipment and hazardous materials used in this protocol.

RECIPES: Please see the end of this protocol for recipes indicated by $<R>$. Additional recipes can be found online at http://cshprotocols.cshlp.org/site/recipes.

\section{Reagents}

Diethylpyrocarbonate (DEPC) $-\mathrm{H}_{2} \mathrm{O}<\mathrm{R}>$

Extracellular saline, RNase-free $(1 \times, \mathrm{pH} 7.3)<\mathrm{R}>$

GFP mRNA template (e.g., from Clontech; see Step 12)

Live/dead cell assay kit (e.g., from Molecular Probes)

Lucifer yellow (Molecular Probes L-6950)

Pollen sample (prepared coverslip; e.g., Carolina 304264)

Primary neurons

\footnotetext{
${ }^{3}$ Correspondence: eberwine@pharm.med.upenn.edu

(C) 2014 Cold Spring Harbor Laboratory Press

Cite this protocol as Cold Spring Harb Protoc; doi:10.1101/pdb.prot072421
} 
J. Lee et al.

For this example, neuron-enriched primary rat hippocampal cultures from E18 embryos were plated at 100,000 per $\mathrm{mL}$ in neurobasal medium (Invitrogen) with B-27 supplement on 12-mm round German Spiegelglas coverslips (Bellco) or gridded coverslips as described in Buchhalter and Dichter (1991).

RNA transcription kit (e.g., Ambion mMESSAGE mMACHINE T7 Kit [Life Technologies AM1344]) RNase AWAY

Equipment

Bioanalyzer (Agilent Technologies G2946CA)

Confocal microscope (e.g., Olympus BX 61 with Prairie Ultima scanner or Zeiss 510meta NLO with manipulator)

Imaging chamber (Warner Instruments 64-0387)

Laser power meter and detector (Melles Griot)

Laser, pulsatile IR (e.g., Chameleon [Coherent Inc.] or Mai-Tai DeepSee [Spectra Physics])

Micropipette, glass (optional; see Step 15)

Micropipette injector system (optional; see Step 15)

\section{METHOD}

\section{Aligning the Multiphoton Beam in the Microscope}

To establish the microscope-specific parameters for phototransfection, a commercially prepared slide of a pollen sample is used to verify the $\mathrm{x}, \mathrm{y}$, and $\mathrm{z}$ positions of the multiphoton beam. Because of the dangers of using a multiphoton laser, all of these procedures should be followed based on institutional laser safety guidelines regarding the use of lasers.

1. Place the pollen coverslip on the confocal microscope stage.

2. Collect $z$-section images.

3. Use the $z$-section images to determine the center of the pollen grain and focus to that location.

4. Center the multiphoton beam in the middle of the image.

This step will vary depending on the microscope in use. For example, if your scope has two independent optical paths for imaging and uncaging, you will require a few more steps to align the imaging pixel and uncaging pixel location. If your microscope uses a single path and shares the same galvanomirror for imaging and uncaging, these steps are not necessary.

5. After removing a lens, place the laser power meter detector in the nosepiece. Measure the power of the multiphoton laser in the back aperture of the lens.

6. Using the designated wavelength and power, irradiate the pollen sample with the beam, and collect $z$ sections to locate the area of photobleaching.

Various laser wavelengths and power levels are used for phototransfection depending on the target cell types and laboratory setups. For this example, we are using $35 \mathrm{~mW}$ at a $720-n m$ wavelength.

Usually, it is easy to identify the photobleached area. However, a high laser power can result in blasting of the pollen or the generation of bubbles. In this case, lower the laser power or the duration of irradiation.

7. Confirm the phototransfection location by comparing the $z$-section images pre- and postbeam irradiation.

The point of phototransfection and the power transmitted by the laser will vary based on the apochromatic and plan correctiveness of the lens. Therefore, the lens should be selected with care and users are advised to consult with the microscope manufacturer for detailed information on choosing the appropriate lens for their systems.

Some microscopes are equipped with a variable collimator lens for IR wavelength. If this is the case, you can manipulate the IR beam focus independently from the imaging wavelength, allowing precise positioning of the $I R$ beam in any z location you choose. Focusing specifically onto the plasma membrane is key to a successful phototransfection. 


\section{Configuring the Parameters for Maximal Cell Viability}

To optimize the power and duration of the IR laser, a cell-type-specific tolerance test is recommended. For the steps below, a coverslip of primary rat hippocampal neurons bathed in $1 \mathrm{~mL}$ of extracellular saline in an imaging chamber was used. Use a membrane-impermeable dye to verify successful phototransfection. For example, 1 mm Lucifer yellow in saline works as a contrast agent, with the cell cytosol appearing dark against a bright extracellular space.

8. Set up frame mode $(512 \times 512)$ confocal imaging with an excitation wavelength of $457 \mathrm{~nm}$. Because the aim of this experiment is to detect a fluorescence signal increase in the cytosol, a high gain setting of the photomultiplier tube is acceptable. Even under optimal conditions, it is often difficult to detect low-level fluorescence changes in frame scan mode because cytosolic dye diffuses from the site of the pore to distal regions. To determine the minimal laser irradiation for maximal cell viability, use a fast line scan, as follows, to help set the optimal configuration.

i. Select a line scan area encompassing a region of bright extracellular space and dark cytosol.

ii. Select the irradiation region on the line scan area and measure the onset of fluorescence signals in the cytosol after irradiation.

iii. After successful dye introduction, store the coverslip in the incubator and monitor cell viability using a live/dead cell assay kit.

9. Aim the beam at the edge of the contrast border using the region-of-interest selection tools in the microscopy software.

10. Turn on the IR beam and, to ensure that pore formation occurs, measure the fluorescence intensity changes from null to bright in the cytosol caused by diffusion of Lucifer yellow (Fig. 1) (Barrett et al. 2006; Sul et al. 2009).

11. To find the optimal conditions for the IR irradiation, test multiple wavelengths (from UV to IR) and durations (microsecond to millisecond). Also test a single irradiation or a train of beams per specific experimental paradigms. To facilitate successful phototransfection, determine the parameters of laser power or irradiation time that cause lethality by immediately filling the cytosol with dye or by bursting the cell and then lowering the power and time of irradiation to a power train that works.

\section{Phototransfection of Neurons with mRNA}

To express target $m R N A$ in a neuron, either a single specific $m R N A$ or multiple $m R N A$ s harvested from culture can be used. In this protocol, GFP mRNA is used as an example. Special precautions are needed to prevent RNase contamination over the course of the experiment. Gloves should be worn and RNase-free materials and technique should be used at all times.

12. Use the RNA transcription kit to synthesize GFP mRNA that is poly-A tailed and capped. Verify the quality of the RNA (e.g., by analysis on a Bioanalyzer).

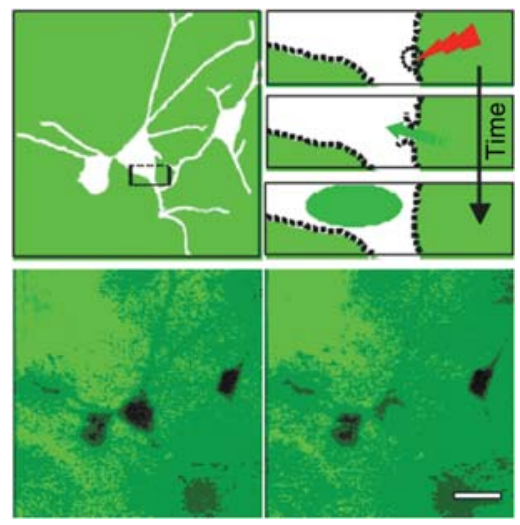

FIGURE 1. Live hippocampal neurons before poration (upper left: diagram, bottom left: live image with Lucifer yellow); note they are not labeled with Lucifer yellow (bottom left). After poration of the center neuron the cell becomes fluorescent around the site of poration showing that the cell has taken up the Lucifer yellow (upper right: illustration, bottom right: live Lucifer yellow image). Also note that the other neurons remain nonfluorescent because photons were not delivered to their processes, demonstrating the spatial control provided by poration. Scale bar, $20 \mu \mathrm{m}$. 
J. Lee et al.

13. Carefully clean the microscope lens and stage area with RNase AWAY.

14. Clean the $40 \times$ water-immersion microscope lens with DEPC -treated $\mathrm{H}_{2} \mathrm{O}$ to remove any residual RNase AWAY. Dry the lens.

15. Place a coverslip of primary rat hippocampal neurons in $1 \mathrm{~mL}$ of extracellular saline containing 10-15 $\mu \mathrm{g}$ of capped GFP mRNA in the imaging chamber that has been prewarmed and maintained at $37^{\circ} \mathrm{C}$.

Alternatively, to increase the local concentration of $m R N A$, use a glass micropipette and injector system to spray additional mRNA at the phototransfection site just before IR beam irradiation. In this method, micropipettes backfilled with RNAs $(200 \mathrm{ng} / \mathrm{mL})$ not only increase the local concentration of mRNA but also reduce the total cost of $m R N A$ preparation.

16. Use two sets of independent galvanometers to allow simultaneous imaging and phototransfection. (For the current example, the following setup was used: Zeiss 510meta NLO with manipulator or Prairie Ultima scanner attached to an Olympus BX61 coupled to a titanium:sapphire laser.)

17. Using the transmitted image on a substage detector, select a target for the beam.

Alternatively, image a small amount of Lucifer yellow diffusion to verify successful phototransfection. Lucifer yellow fluorescence signal in the extracellular space also can serve as an indicator of the highest RNA concentration surrounding the neuron.

18. Deliver $\sim 100$-fsec laser beam pulses ( $\sim 80 \mathrm{MHz}$ repetition rate) for $5 \mathrm{msec}$ at a power of $35 \mathrm{~mW}$ at the back aperture of lens to a $4 \times 4$ pixel region of interest (ROI) using random scanning of pixels to make a transient pore in the cell membrane.

Approximately $80 \%$ of the power is transmitted to the focused spot. Beam size should be diffraction limited. The interval between each cell is $5 \mathrm{~ms}$. These conditions are guidelines only. The duration and power need to be optimized for each cell type and cell culturing method. As an alternative, instead of relatively long millisecond irradiation times, duration can be reduced to microseconds by using a train of pulses.

19. After phototransfection of GFP mRNA, replace the saline with fresh RNase-free saline and image the cells to detect GFP protein translated from the phototransfected mRNA (Fig. 2; Barrett et al. 2006; Sharma et al. 2010).

20. If the desired expression level has not been reached, repeat the phototransfection.

Phototransfection can be repeated multiple times using various time intervals ranging from hours to days to reach a desired expression level. Sterile technique becomes increasingly critical as the intervals between phototransfection sessions increase.

Targeted transfection in designated cells within a population is important for understanding the role of single cells in a system. Phototransfection is a highly specific cellular compartment-directed mRNA
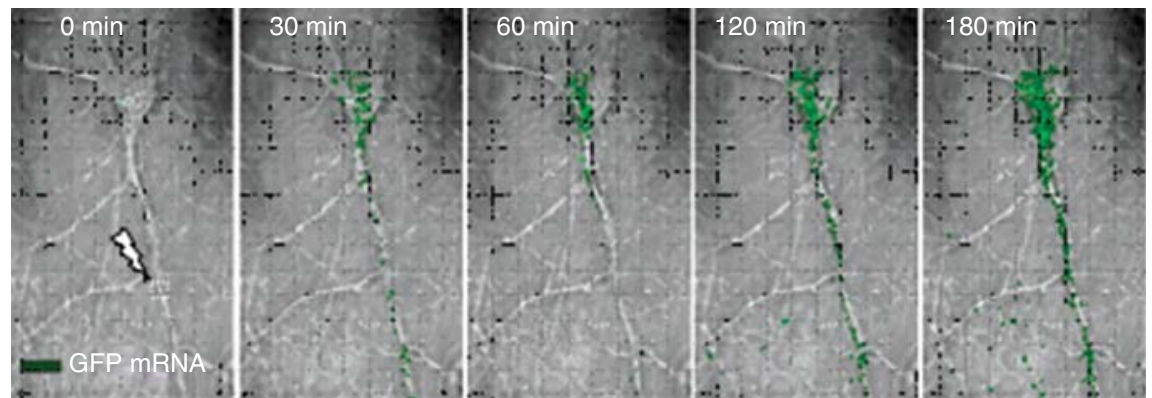

FIGURE 2. Neurons were phototransfected in the presence of $10 \mu \mathrm{g} / \mathrm{mL}$ of GFP mRNA. The box outlines the area of phototransfection. Differential interference contrast (DIC) images showing a neuron before phototransfection and at indicated times after phototransfection are overlayed with the GFP fluorescent signal (green). Scale bar, $20 \mu \mathrm{m}$. 
transfection method. For example, one can express multiple transcripts in differing amounts in distinct cells or compartments within the same cells. Because phototransfection requires only standard imaging instruments, it is straightforward to adopt this method in laboratories that already routinely perform confocal microscopy. The feasibility of this technique has been showed by a number of laboratories using different laser wavelengths and configurations for different cell types (Stevenson et al. 2010).

\section{RECIPES}

\section{DEPC $-\mathrm{H}_{2} \mathrm{O}$}

Mix $1 \mathrm{~mL}$ of DEPC (diethyl pyrocarbonate) with $1000 \mathrm{~mL}$ of water in a screw-cap glass bottle. Incubate for $\sim 2 \mathrm{~h}$ at room temperature in a fume hood with occasional swirling. Autoclave. Store at room temperature for up to $12 \mathrm{mo}$.

Extracellular Saline, RNase-Free (1×, pH 7.3)

\begin{tabular}{lcc} 
Reagent & $\begin{array}{c}\text { Quantity }(\mathrm{g}) \\
\text { (for } 500 \mathrm{~mL})\end{array}$ & $\begin{array}{c}\text { Final concentration } \\
(\mathrm{mm})(1 \times)\end{array}$ \\
\hline $\mathrm{NaCl}$ & 4.091 & 140 \\
$\mathrm{KCl}$ & 0.201 & 5.4 \\
$\mathrm{CaCl}_{2}$ & 0.147 & 2.0 \\
$\mathrm{MgSO}_{4}$ & 0.120 & 10 \\
$\mathrm{HEPES}$ & 1.191 & 5.0 \\
Glucose & 1.442 & 10 \\
Prepare extracellular saline under RNase-free conditions and with $\mathrm{DEPC}-\mathrm{H}_{2} \mathrm{O}$. &
\end{tabular}

\section{REFERENCES}

Barrett LE, Sul JY, Takano H, Van Bockstaele EJ, Haydon PG, Eberwine JH. 2006. Region-directed phototransfection reveals the functional significance of a dendritically synthesized transcription factor. Nat Methods 3: 455-460.

Buchhalter JR, Dichter MA. 1991. Electrophysiological comparison of pyramidal and stellate nonpyramidal neurons in dissociated cell culture of rat hippocampus. Brain Res Bull 26: 333-338.

Sul JY, Sharma A, Eberwine J, Haydon PG. 2009. Nucleic acid introduction into primary neurons and glia. Encyclopedia of neuroscience, pp. 1283-1286. Academic, Oxford.
Sharma A, Callahan LM, Sul JY, Kim TK, Barrett L, Kim M, et al. 2010. A neurotoxic phosphoform of Elk-1 associates with inclusions from multiple neurodegenerative diseases. PloS ONE 5: e9002. doi: 10.1371/journal.pone.0009002.

Stevenson DJ, Gunn-Moore FJ, Campbell P, Dholakia K. 2010. Single cell optical transfection. J R Soc Interface 7: 863-871. 


\section{Single Cell/Cellular Subregion-Targeted Phototransfection}

Jaehee Lee, Jai-Yoon Sul and James H. Eberwine

Cold Spring Harb Protoc; doi: 10.1101/pdb.prot072421

\begin{tabular}{cc}
$\begin{array}{r}\text { Email Alerting } \\
\text { Service }\end{array}$ & Receive free email alerts when new articles cite this article - click here. \\
\hline $\begin{array}{r}\text { Subject } \\
\text { Categories }\end{array}$ & $\begin{array}{c}\text { Browse articles on similar topics from Cold Spring Harbor Protocols. } \\
\text { Confocal Microscopy (114 articles) } \\
\text { DNA Delivery/Gene Transfer (344 articles) } \\
\text { DNA Delivery/Gene Transfer, general (341 articles) } \\
\text { Non-Viral Methods (226 articles) }\end{array}$ \\
\hline
\end{tabular}

